\title{
L4 and L5 Spondylectomy for En Bloc Resection of Giant Cell Tumor and Review of the Literature
}

\author{
David R. Santiago-Dieppa ${ }^{1} \quad$ Lee S. Hwang ${ }^{1} \quad$ Ali Bydon $^{1} \quad$ Ziya L. Gokaslan ${ }^{1}$ Edward F. McCarthy ${ }^{2}$ \\ Timothy F. Witham ${ }^{1}$ \\ 1 Department of Neurosurgery, The Johns Hopkins University School of \\ Medicine, Baltimore, Maryland, United States \\ 2 Department of Pathology, The Johns Hopkins University School of \\ Medicine, Baltimore, Maryland, United States \\ Address for correspondence Timothy F. Witham, MD, Department of \\ Neurosurgery, The Johns Hopkins Hospital, Meyer 7-109, 600 North \\ Wolfe Street, Baltimore, MD 21287, United States \\ (e-mail: twitham2@jhmi.edu).
}

\begin{abstract}
Keywords

- giant cell tumor

- osteoclastoma

- spondylectomy

- lumbar spine

- lumbopelvic reconstruction

Study Design Case report and review of the literature.

Objective We present the case of a two-level lumbar spondylectomy at L4 and L5 for en bloc resection of a giant cell tumor (GCT) and lumbopelvic reconstruction.

Methods A 58-year-old woman presented with a 7-month history of progressive intractable back and leg pain secondary to a biopsy-proven Enneking stage III GCT of the $L 4$ and $L 5$ vertebrae. The patient underwent a successful $L 4-L 5$ spondylectomy and lumbopelvic reconstruction using a combined posterior and anterior approach over two operative stages.

Results Postoperative complications included a deep wound infection and a cerebrospinal fluid leak; however, following surgical debridement and long-term antibiotic treatment, the patient was neurologically intact with minimal pain and there was no evidence of tumor recurrence or instrumentation failure at more than 2 years of follow-up.

Conclusion Spondylectomy that achieves en bloc resection is a viable and effective treatment option that can be curative for Enneking stage III GCTs involving the lower lumbar spine. The lumbosacral junction represents a challenging anatomic location for spinal reconstruction after spondylectomy with unique technical considerations.
\end{abstract}

\section{Introduction}

Giant cell tumors (GCTs) are biologically unpredictable primary neoplasms of bone. ${ }^{1}$ GCTs are reported to comprise only $5 \%$ of all primary bone tumors in adults and most commonly affect the appendicular skeleton. ${ }^{1,2}$ Only 2 to $4 \%$ of all GCTs afflict the mobile spine, ${ }^{2-4}$ and those affecting the lower lumbar spine (LLS) represent a significantly smaller proportion of this population. Furthermore, in contrast to GCTs of the appendicular skeleton, GCTs of the mobile spine carry a significantly worse prognosis due to the delicate and complex neurologic structures that can be easily damaged by the aggressive osteolytic nature of these tumors. Recent evidence suggests that spondylectomy achieving en bloc resection of GCTs may promote disease-free survival relative to intrale-

received

December 23, 2013

accepted

July 8,2014

sional resections, ${ }^{4,5}$ especially for Enneking stage III GCTs of the mobile spine. ${ }^{6}$

Although many techniques for spondylectomy exist, the most important goal of this procedure in the oncologic setting should be en bloc resection of the tumor, which is defined as removal of the entire vertebra(e) with the tumor intact and encompassed by variable margins of disease-free tissue. ${ }^{7}$ However, despite being potentially curative, complete spondylectomy of multiple levels of LLS for GCT is a complex and technically challenging procedure that requires multidisciplinary expertise and careful presurgical planning to limit potential morbidity to the patient.

In this report, we present the unique and challenging case of a 58-year-old woman who was found to have a primary isolated GCT involving the L4 and L5 vertebral bodies. In

(c) 2014 Georg Thieme Verlag KG Stuttgart · New York
DOI http://dx.doi.org/ 10.1055/s-0034-1387804. ISSN 1663-7976. 
addition, we describe, in detail, the two-staged technique of a combined posterior and anterior approach to total L4-L5 spondylectomy as well as reconstruction of the lumbopelvic junction. Adjuvant therapy has not been necessary after more than 2 years of follow-up. We will also review the literature on the treatment of GCTs of the mobile spine.

\section{Case Report}

\section{Clinical Presentation}

A 58-year-old woman with a medical history significant for thyroid disease presented to her primary care provider with a 3-month history of constant back pain localized to the lower lumbar region. Despite some minor improvement in pain with conservative medical management, the patient developed severe left side predominant sciatica in an S1 distribution. She reported weakness with standing and ambulation but denied sensory, bowel, or bladder changes. T2-weighted MRI showed a low-signal lesion at L5 that extended across the L4-L5 disk space into the L4 vertebral body which intensely enhanced on T1-weighted postcontrast images ( - Fig. 1A-D). In addition, there was loss of vertebral column height at L5, mild retropulsion of the bone into the spinal canal, and bilateral narrowing of the L5 neuroforamina with neural impingement. Computer tomography (CT) disclosed a lytic vertebral lesion at L4 and L5 correlating with the MRI (-Fig. 2A, B). The patient subsequently underwent a CTguided biopsy of the lesion that demonstrated an Enneking stage III GCT of bone.

The patient was referred to our service for consideration of surgical management. Neurological examination revealed 5/5 strength in all muscle groups of the lower extremities. Sensation was intact to light touch.

Due to the unpredictable nature of GCT and its strong propensity for local recurrence, a spondylectomy that achieved en bloc resection of the tumor followed by lumbopelvic reconstruction was recommended to optimize the patient's chance of disease-free survival. Such a procedure would be executed in two stages.

\section{Operative Procedure}

\section{Stage 1}

The day prior to the first stage of the operation, the patient underwent bilateral transarterial embolization of the L4 and L5 radicular arteries using coils. A midline posterior approach was performed with bilateral exposure from L2-S2. Pedicle screw instrumentation (DePuy Expedium, Ryanham, Massachusetts, United States) was placed at L2, L3, and S1 bilaterally. S2 alar-iliac (S2AI) screws were also placed via the S2 pedicle approach on the right. A separate posterior superior iliac spine approach was required on the left after the S2AI approach could not be successfully performed.

L3 laminectomy and medial L3-L4 facetectomy were performed with a bone scalpel (Aesculap, Tuttlingen, Germany). Complete en bloc resection of the posterior elements of L4-L5 was complicated by a greater-than-predicted collapse of L5; therefore, after en bloc removal of the L4 and L5
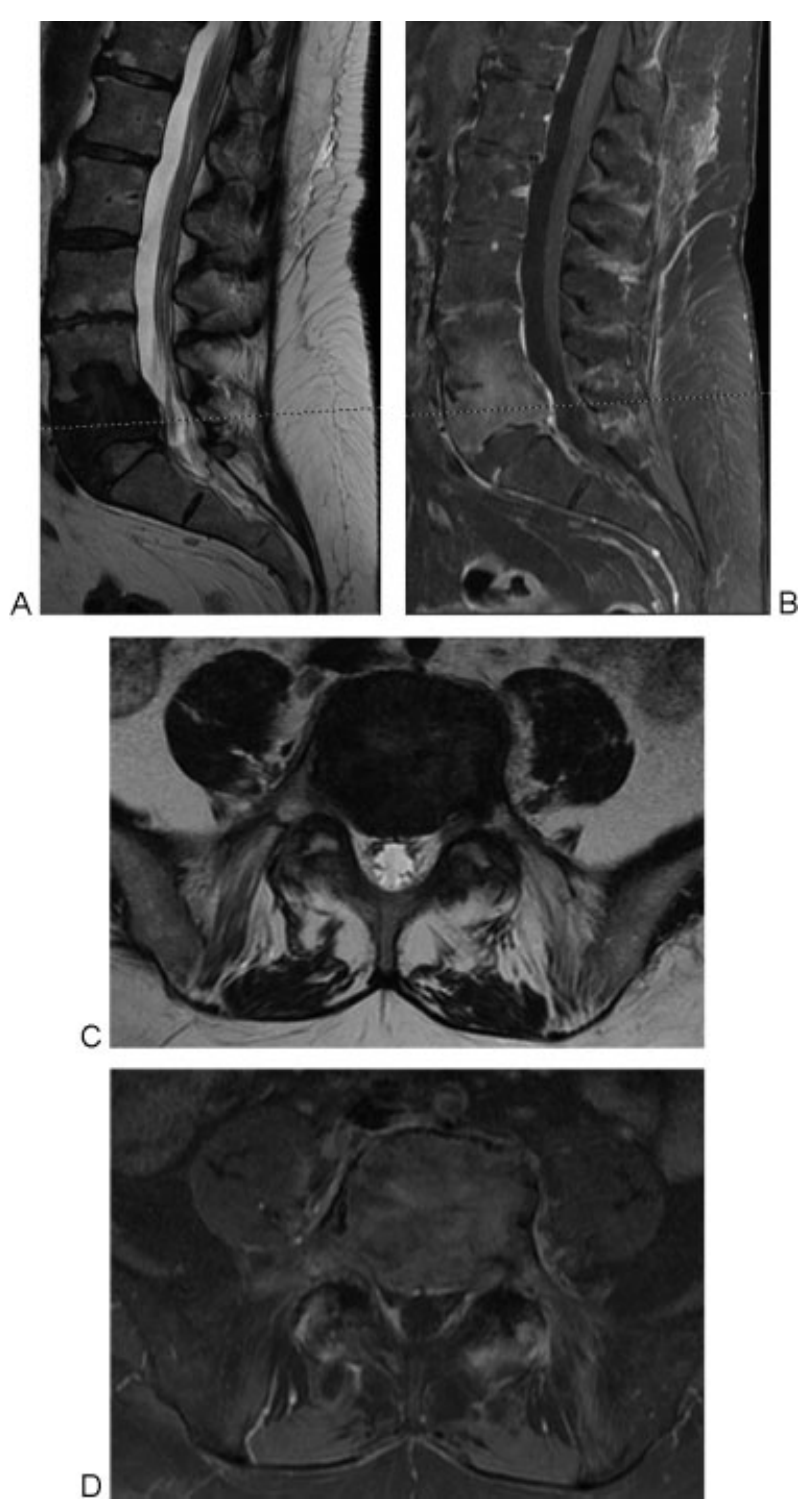

Fig. 1 Preoperative imaging. (A) T2-weighted sagittal MRI; (B) T1weighted postcontrast sagittal MRI; (C) T2-weighted axial MRI; (D) T1weighted postcontrast axial MRI. MRI, magnetic resonance imaging.

lamina, a piecemeal decompression was performed for the bilateral resection of the L3-L4 and L4-L5 facet joints to protect the nerve roots.

The nerve roots of L3-L5 were carefully identified and dissected bilaterally beyond the pedicle to the level of the lumbar plexus to ensure complete mobilization. After bilateral removal of the pedicles at L4 and L5 followed by dissection and mobilization of the ventral aspect of the thecal sac, partial discectomies at L3-L4 and L5-S1 were performed to allow implantation of the Tomita saws for use during the anterior stage of the surgery.

Two 5.5-mm titanium rods were contoured to the lumbar lordosis and secured to the pedicle screws of L2-S1 and S2 on the right, and from L2-S1 on the left with an offset spacer bridging to the left iliac crest screw. A cross-link was placed at the L4-L5 level and then Songer cables were passed anteriorly along the L4 segment for eventual attachment to the cage of the anterior construct. 

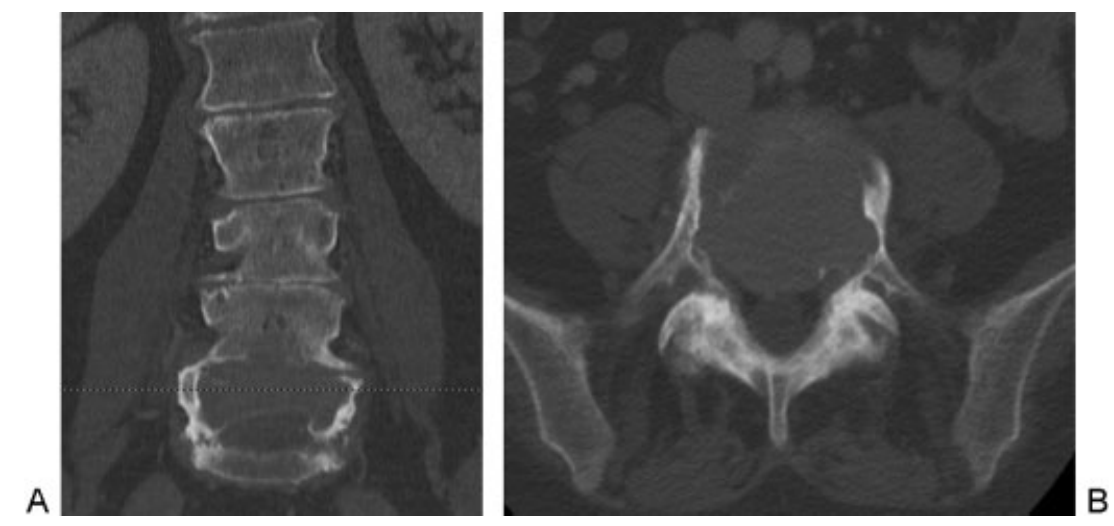

Fig. 2 Preoperative CTscan, coronal (A) and axial (B) views at L5. CT-guided biopsy disclosed giant cell tumor of bone. CT, computed tomography.

\section{Stage 2}

A retroperitoneal dissection was performed by a vascular surgeon for mobilization of the aorta, vena cava, and iliac vessels to achieve wide anterior exposure of the L3-S1 segments.

After mobilization of the vessels, dissection and exposure of the L4 and L5 vertebrae as well as the sacral promontory were performed. Then, the psoas muscle was dissected from the anterolateral aspects of L4 and L5. The Tomita saws, placed in the L3-L4 and L5--S1 disk spaces during the first stage of the operation, were successfully identified and brought anteriorly to attach the handles. Carefully through direct visualization of the vasculature, the disk spaces at L3L4 and L4-L5 were transected, allowing for the delivery of the L4-L5 vertebral segments en bloc (-Fig. 3A).
Anterior lumbosacral reconstruction using a VLIFT titanium cage (Stryker, Kalamazoo, Michigan, United States) was sized to span the L3-S1 gap. End caps were selected based on preoperative imaging (-Fig. 3B). The two Songer cables were fastened to the cage and tightened to ensure anchoring to the posterior hardware construct. An intraoperative X-ray was obtained to confirm proper positioning of the cage. Anterior reconstruction of the spinal column was completed by installing an anterior tension band (ATB) plate (Synthes, West Chester, Pennsylvania, United States) that spanned $101 \mathrm{~mm}$ in length from L3 to S1 (-Fig. 3C). A polytetrafluoroethylene graft (W.L. Gore \& Associates, Inc., Elkton, Maryland, United States) was placed between the major vessels and the instrumentation for the theoretical benefit of preventing scarring and adhesions in addition to maintaining a dissection plane in

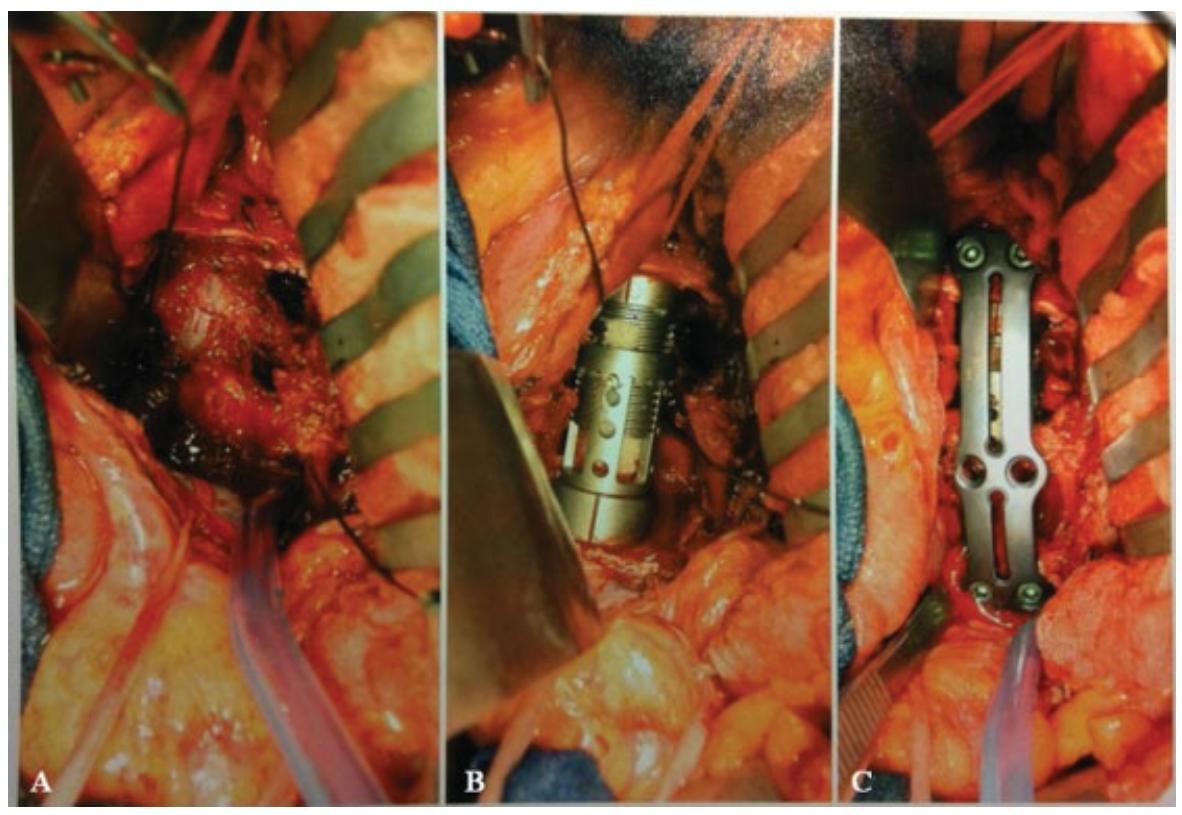

Fig. 3 Intraoperative photographs demonstrating the anterior stage of the L4 and L5 en bloc spondylectomy. (A) Photograph obtained after L3L4 and L5-S1 discectomy completion and en bloc resection of the L4 and L5 vertebral bodies. (B) Photograph acquired after the placement of a distractible titanium cage between the $\mathrm{L} 3$ vertebral body and the sacrum. (C) Photograph demonstrating placement of an anterior tension band plate. 


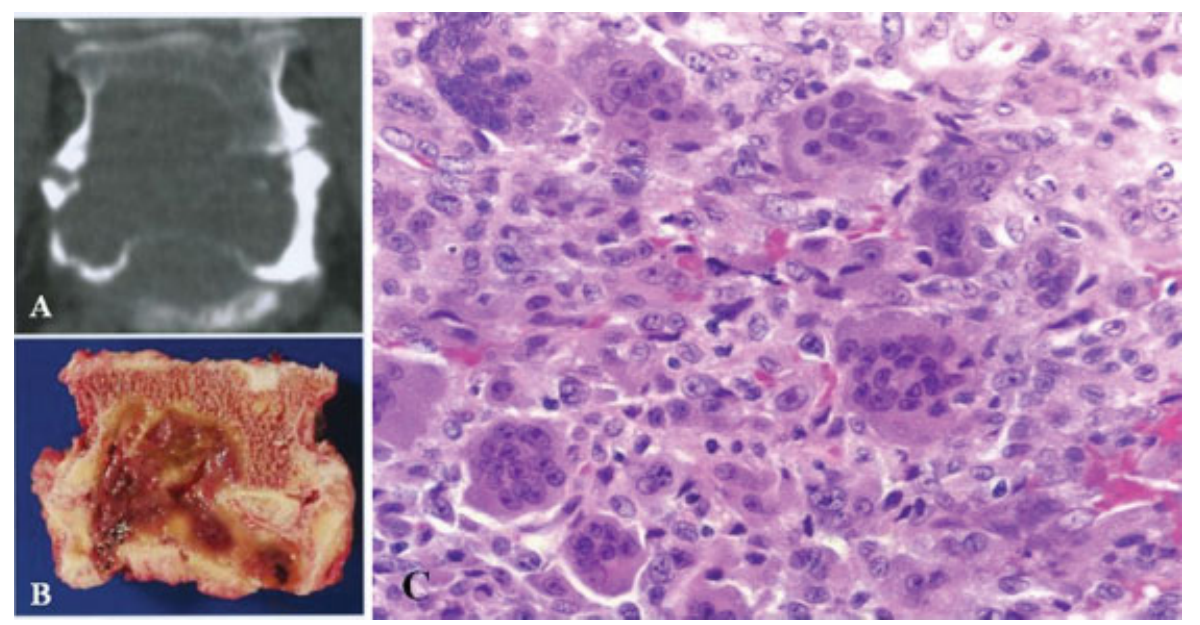

Fig. 4 Pathology. (A) Preoperative CT scan showing L4-L5 segments. (B) Photograph demonstrating the gross specimen of the L5-L4 vertebral body after en bloc resection. (C) Photomicrograph of the hematoxylin and eosin-stained sections demonstrating giant cells. CT, computed tomography. (Figs. 4B, C reprinted with permission from Martin C, McCarthy EF. Giant cell tumor of the sacrum and spine: series of 23 cases and a review of the literature. lowa Orthop J 2010;30:69-75.)

case of revision surgery. There were no immediate complications from the procedure, and the patient remained neurologically intact.

\section{Postoperative Course}

Pathology confirmed the diagnosis of an Enneking stage III GCT, and the margins were found to be negative ( - Fig. 4A-C). The patient was discharged to rehab on hospital day 14 . On postoperative day 16 , dehiscence and serous drainage from the inferior aspect of the posterior wound was noted. Due to concern for a possible deep wound infection, the patient was taken back to the OR, and the wound was reopened, irrigated, and debrided. Operative cultures obtained later revealed a complicated deep wound infection with corynebacterium for which the patient was treated with an extended course of vancomycin and cefepime. Three days after the debridement surgery, the patient was found to have a cerebrospinal fluid leak, which was successfully treated with a temporary lumbar drain. At 28 months of follow-up, the patient was neurologically intact and ambulatory without any evidence of GCT recurrence or instrumentation failure ( - Fig. 5A, B).

\section{Discussion}

GCTs, also known as osteoclastomas, are histologically characterized by a hypercellular field consisting of multinucleated giant cells uniformly dispersed among a population of round to ovoid mononuclear cells-which are the cells thought to be neoplastic. ${ }^{1-3}$ Although classically defined in the literature as benign primary neoplasms of bone, this term can be misleading because they are aggressively osteolytic in nature and can cause substantial local soft tissue destruction. Furthermore, although the tumor is devoid of malignant characteristics, they are known to metastasize to the lungs in up to $13.5 \%$ of cases involving the spine ${ }^{8}$ and can occasionally undergo sarcomatous transformation ${ }^{1,3,9}$ with an incidence as high as $2 \%^{3}$ The majority of morbidity experienced by patients with GCTs of the mobile spine results from the osteolytic destruction and structural compromise of substantial portions of the vertebra(e), which can lead to intractable back pain, neurological deficits, and disability.

The present work has evolved from two of our previously reported cases. ${ }^{10,11}$ In the first report, we described a patient with a GCT involving the L5 vertebral body. This patient underwent a combined posterior-anterior approach for en bloc resection of the tumor followed by lumbopelvic reconstruction that was accomplished posteriorly by pedicle and iliac screws and anteriorly by placement of a distractible titanium cage and ATB plate spanning L4-S1. The second report described a patient who presented with a chordoma of L4 that extended superiorly to L3 and inferiorly to L5. Similar to the first case, we utilized a combined posterior-anterior approach to achieve a three-level spondylectomy that resulted in en bloc resection of the patient's chordoma.
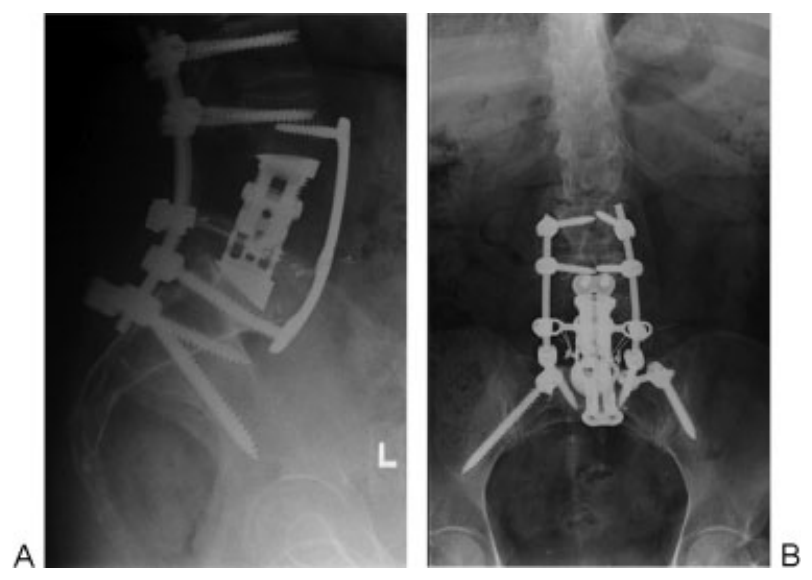

Fig. 5 Postoperative imaging studies. (A) AP and (B) lateral plain radiographs obtained 2 years after the surgery revealing the spinal alignment, anterior reconstruction, and posterior stabilization. AP, anteroposterior. 
Lumbopelvic reconstruction was achieved posteriorly by the placement of pedicle and iliac screws and anteriorly by placement of a distractible titanium cage. The cage was also wired to the posterior construct to prevent anterior displacement.

Because GCTs of the mobile spine are rare, there are only a few case series ${ }^{4-6}$ and one systematic review ${ }^{12}$ that address treatment outcomes. Furthermore, only a few groups have reported cases of spondylectomy for en bloc resection of any neoplasm involving the LLS, ${ }^{11,13-18}$ and currently there are only three reported cases of spondylectomy for en bloc resection of any neoplasm involving two or more levels of the LLS. ${ }^{5,10}$ Despite their rarity, it is imperative that the spine surgeon know how to effectively treat GCTs of the spine. From an evidence-based perspective, there are no prospective randomized studies that evaluate various treatments; however, there is evidence in the literature that spondylectomy for en bloc resection is associated with the best prognosis of long-term, disease-free survival. ${ }^{4,11,12,18-24}$ When GCTs of the mobile spine are resected in an en bloc fashion, the prognosis of disease-free survival has been reported to be as high as $92.3 \%{ }^{25}$ Furthermore, and in stark contrast to en bloc resection, GCTs of the spine treated through intralesional surgeries are associated with disease-free survival rates of $72.2 \%{ }^{25}$ Therefore, we believe that spondylectomy achieving en bloc resection with reconstruction is the superior treatment option for GCTs of the spine.

Adjuvant and alternative treatments to surgery for GCTs of the spine do exist. GCTs can be radiosensitive, and radiotherapy is associated with long-term, local control rates ranging from 60 to $84 \%{ }^{26}$; however, lower rates are reported for tumors larger than $8.5 \mathrm{~cm}$ and for locally recurrent disease. ${ }^{27}$ Using megavoltage radiation therapy for axial or inoperable GCTs remains controversial, although one study reported $85 \%$ of such tumors did not progress after megavoltage radiation with no malignant transformation during long-term followup. ${ }^{28}$ Radiation therapy is often used for local recurrence and following incomplete excision; however, this supplementary treatment is not always successful and is associated with a long-term risk of developing radiation-induced sarcoma. ${ }^{29}$

Because GCTs of the mobile spine are characteristically vascular tumors, we strongly recommend preoperative arterial embolization of the segmental arteries corresponding to the anticipated level(s) of resection to reduce intraoperative blood loss. Arterial embolization has also been assessed as monotherapy for GCTs of the mobile spine. Hosalkar et al reported that therapeutic embolization was successful in seven of nine patients with sacral GCTs, without adjuvant radiation or surgical intervention, during 8 years of follow-up care. ${ }^{30}$ On the other hand, two patients required radiation therapy with successful outcome in one and fatal pulmonary metastases in the other. ${ }^{19}$ In addition, Lin et al demonstrated a $31 \%$ risk of recurrence at 10 years after treatment with therapeutic embolization. ${ }^{31}$

Chemotherapy is generally not considered a standard therapeutic approach for GCTs of the mobile spine. Interferon- $\alpha$ may be utilized for more aggressive disease but is associated with significant side effects. ${ }^{32,33}$ There are no randomized studies that demonstrate the efficacy of interferon on GCTs. In addition, several clinical reports suggest that bisphosphonates may yield symptomatic benefit and local disease control even for prolonged periods. ${ }^{34}$ However, there are no reports of antitumor activity following preoperative treatment, and the role of bisphosphonates in a clinical setting remains unclear. Furthermore, Denosumab, a human monoclonal antibody against RANKL, has been shown to significantly reduce the number of giant cells upon histologic evaluation as well as halt radiographic progression of the lesion. ${ }^{35}$ Denosumab is approved only for treatment of osteoporosis in the United States, and a global clinical trial protocol is available to assess its efficacy on advanced or selected resectable GCTs. ${ }^{36}$ Its use as adjuvant therapy is currently under investigation, and whether it can be used to decrease local tumor recurrence is unknown at this time.

In addition to obtaining wide or marginal resection of a tumor, the goal of spondylectomy is to preserve as much neurological function as possible and reconstruct the spine to achieve axial stability and mechanical function. ${ }^{10}$ Reconstruction of the lumbosacral junction after a two-level spondylectomy of L4-L5 is immensely challenging because this region plays a foundational role in transmitting compressive, sheer, torsional, and bending forces to the pelvis. ${ }^{10}$ In this patient, we used a distractible titanium cage as the primary weightbearing structure.

Preoperative imaging allowed us to measure the angulation required by the rostral and caudal caps of the cage. Preoperative selection of the proper caps allows one to optimally position the cage about a sagittal axis of rotation, which, in conjunction with the posterior construct rods, aids in preserving the lordosis of lumbar spine. From a biomechanical perspective, maintaining the lordosis of the lumbar spine is crucial in preserving the long-term function of the spine as a whole. To prevent forward displacement of the cage, an ATB plate and wires anchored to the rods of the posterior instrumentation were used in this patient. At this time, there is no literature specifically addressing the longevity of such a construct.

Performing spondylectomy involving one or more vertebral levels to resect spinal neoplasms in an en bloc fashion is technically challenging especially in the LLS. The potential morbidity associated with spondylectomies for en bloc resection of tumors is well documented. In the largest and most recent retrospective observational study, Boriani et al reported that $35.1 \%$ of patients who underwent spondylectomy with en bloc resection of tumor experienced at least one complication. ${ }^{25}$ This study also reported that the rate of complications in patients who had prior treatment of their tumors was $45.7 \%$, while the rate of complications in patients undergoing first-time treatment was $31.3 \%$. In addition to the higher rate of complications in the repeat-treatment group, this study also reported that complication rates were higher with resection of two or more vertebral levels. Despite the risks, multilevel resections are necessary if the tumor has invaded adjacent levels, and en bloc resection is desired. Finally, Borani et al reported a lower rate of complications associated with a posterior-only approach to spondylectomy; however, such an approach can be performed 
only in certain situations ${ }^{31,37}$ and is not feasible in patients who require en bloc resection of tumors involving the lumbar spine. ${ }^{5,10,11,38-40}$ A combined posterior and anterior surgical approach to spondylectomy in the lumbar spine is usually the preferred procedure. ${ }^{5,38-41}$ We also advocate this approach in the LLS because of optimal visualization of vital anatomic structures near the vertebral bodies and a far greater likelihood of preserving the lumbosacral nerve roots as they exit the spinal column anteriorly.

\section{Conclusion}

Spondylectomy that achieves en bloc resection followed by lumbosacral reconstruction is a feasible and effective treatment option that can be curative for primary GCTs involving multiple levels of the LLS. Multilevel spondylectomy at the lumbosacral junction that achieves disease-free margins is a complex and technically challenging procedure that requires a multidisciplinary team and careful preoperative consideration of the patient's medical comorbidities.

\author{
Disclosures \\ David R. Santiago-Dieppa, none \\ Lee S. Hwang, none \\ Ali Bydon, none \\ Ziya L. Gokaslan, none \\ Edward F. McCarthy, none \\ Timothy F. Witham, none
}

\section{References}

1 Reid R, Banerjee SS, Sciot R. Giant cell tumor. In: Fletcher CD, Unni KK, Mertens F, eds. World Health Organization Classification of Tumours. Pathology and Genetics. Tumours of Soft Tissue and Bone. Lyon, France: IARC Press; 2002:310-312

2 Turcotte RE. Giant cell tumor of bone. Orthop Clin North Am 2006; 37(1):35-51

3 Mendenhall WM, Zlotecki RA, Scarborough MT, Gibbs CP, Mendenhall NP. Giant cell tumor of bone. Am J Clin Oncol 2006;29(1): 96-99

4 Martin C, McCarthy EF. Giant cell tumor of the sacrum and spine: series of 23 cases and a review of the literature. Iowa Orthop J 2010;30:69-75

5 Kawahara N, Tomita K, Murakami H, Demura S, Yoshioka K, Kato S. Total en bloc spondylectomy of the lower lumbar spine: a surgical techniques of combined posterior-anterior approach. Spine (Phila Pa 1976) 2011;36(1):74-82

6 Boriani S, Bandiera S, Casadei R, et al. Giant cell tumor of the mobile spine: a review of 49 cases. Spine (Phila Pa 1976) 2012;37(1): E37-E45

7 Boriani S, Weinstein JN, Biagini R. Primary bone tumors of the spine. Terminology and surgical staging. Spine (Phila Pa 1976) 1997;22(9):1036-1044

8 Donthineni R, Boriani L, Ofluoglu O, Bandiera S. Metastatic behaviour of giant cell tumour of the spine. Int Orthop 2009;33(2):497-501

9 Campanacci M, Baldini N, Boriani S, Sudanese A. Giant-cell tumor of bone. J Bone Joint Surg Am 1987;69(1):106-114

10 Clarke MJ, Hsu W, Suk I, et al. Three-level en bloc spondylectomy for chordoma. Neurosurgery 2011;68(2, Suppl Operative) 325-333, discussion 333
11 Gallia GL, Sciubba DM, Bydon A, et al. Total L-5 spondylectomy and reconstruction of the lumbosacral junction. Technical note. J Neurosurg Spine 2007;7(1):103-111

12 Harrop JS, Schmidt MH, Boriani S, Shaffrey CI. Aggressive "benign" primary spine neoplasms: osteoblastoma, aneurysmal bone cyst, and giant cell tumor. Spine 2009;34(22, Suppl)S39-S47

13 Abe E, Sato K, Tazawa H, et al. Total spondylectomy for primary tumor of the thoracolumbar spine. Spinal Cord 2000;38(3): 146-152

14 Boriani S, De Iure F, Bandiera S, et al. Chondrosarcoma of the mobile spine: report on 22 cases. Spine (Phila Pa 1976) 2000; 25(7):804-812

15 Detwiler PW, Porter RW, Crawford NR, Apostolides PJ, Dickman CA. Lumbosacral junction fixation and fusion after complete L-5 spondylectomy. Case report. Neurosurg Focus 1999;7(6):e3

16 Heary RF, Vaccaro AR, Benevenia J, Cotler JM. "En-bloc" vertebrectomy in the mobile lumbar spine. Surg Neurol 1998;50(6): 548-556

17 Marmor E, Rhines LD, Weinberg JS, Gokaslan ZL. Total en bloc lumbar spondylectomy. Case report. J Neurosurg 2001;95(2, Suppl)264-269

18 Shimada Y, Hongo M, Miyakoshi N, et al. Giant cell tumor of fifth lumbar vertebrae: two case reports and review of the literature. Spine J 2007;7(4):499-505

19 Chi JH, Sciubba DM, Rhines LD, Gokaslan ZL. Surgery for primary vertebral tumors: en bloc versus intralesional resection. Neurosurg Clin N Am 2008;19(1):111-117

20 Fidler MW. Surgical treatment of giant cell tumours of the thoracic and lumbar spine: report of nine patients. Eur Spine J 2001;10(1): 69-77

21 Hart RA, Boriani S, Biagini R, Currier B, Weinstein JN. A system for surgical staging and management of spine tumors. A clinical outcome study of giant cell tumors of the spine. Spine (Phila Pa 1976) 1997;22(15):1773-1782, discussion 1783

22 Sanjay BK, Sim FH, Unni KK, McLeod RA, Klassen RA. Giant-cell tumours of the spine. J Bone Joint Surg Br 1993;75(1):148-154

23 Stener B, Johnsen OE. Complete removal of three vertebrae for giant-cell tumour. J Bone Joint Surg Br 1971;53(2):278-287

24 Tomita K, Kawahara N, Baba H, Tsuchiya H, Fujita T, Toribatake Y. Total en bloc spondylectomy. A new surgical technique for primary malignant vertebral tumors. Spine (Phila Pa 1976) 1997;22(3): 324-333

25 Boriani S, Bandiera S, Donthineni R, et al. Morbidity of en bloc resections in the spine. Eur Spine J 2010;19(2):231-241

26 Ruka W, Rutkowski P, Morysiński T, et al. The megavoltage radiation therapy in treatment of patients with advanced or difficult giant cell tumors of bone. Int J Radiat Oncol Biol Phys 2010;78(2): 494-498

27 Caudell JJ, Ballo MT, Zagars GK, et al. Radiotherapy in the management of giant cell tumor of bone. Int J Radiat Oncol Biol Phys 2003; 57(1):158-165

28 Chakravarti A, Spiro IJ, Hug EB, Mankin HJ, Efird JT, Suit HD. Megavoltage radiation therapy for axial and inoperable giantcell tumor of bone. J Bone Joint Surg Am 1999;81(11): 1566-1573

29 Leggon RE, Zlotecki R, Reith J, Scarborough MT. Giant cell tumor of the pelvis and sacrum: 17 cases and analysis of the literature. Clin Orthop Relat Res 2004;(423):196-207

30 Hosalkar HS, Jones KJ, King JJ, Lackman RD. Serial arterial embolization for large sacral giant-cell tumors: mid- to long-term results. Spine (Phila Pa 1976) 2007;32(10):1107-1115

31 Lin PP, Guzel VB, Moura MF, et al. Long-term follow-up of patients with giant cell tumor of the sacrum treated with selective arterial embolization. Cancer 2002;95(6):1317-1325

32 Wei F, Liu X, Liu Z, et al. Interferon alfa-2b for recurrent and metastatic giant cell tumor of the spine: report of two cases. Spine (Phila Pa 1976) 2010;35(24):E1418-E1422 
33 Kaban LB, Troulis MJ, Wilkinson MS, Ebb D, Dodson TB. Adjuvant antiangiogenic therapy for giant cell tumors of the jaws. J Oral Maxillofac Surg 2007;65(10):2018-2024, discussion 2024

34 Arpornchayanon O, Leerapun T. Effectiveness of intravenous bisphosphonate in treatment of giant cell tumor: a case report and review of the literature. J Med Assoc Thai 2008;91(10):1609-1612

35 Thomas D, Henshaw R, Skubitz K, et al. Denosumab in patients with giant-cell tumour of bone: an open-label, phase 2 study. Lancet Oncol 2010;11(3):275-280

36 ClinicalTrials.gov (A service of the U.S. National Institutes of Health). Safety and efficacy study of denosumab in patients with recurrent or unresectable giant cell tumor of bone (2008). Available at: http://www.clinicaltrials.gov/ct2/show/NCT00680992? term=denosumab\&cond $=$ Giant + cell + tumor + of + bone\&rank $=1$. Accessed April 13, 2013

37 Hsieh PC, Li KW, Sciubba DM, Suk I, Wolinsky JP, Gokaslan ZL. Posterior-only approach for total en bloc spondylectomy for malig-

\section{Editorial Perspective}

EBSJ appreciates the interesting case presentation by our colleagues from The Johns Hopkins University in Baltimore, United States and the thoughtful review by Dr. Lazary from Budapest, Hungary.

The key insights gained from these two contributions can probably be summarized best as follows:

1. Proper diagnosis and staging of such lesions is preferably performed at centers with significant spine neoplasia experience and in-house multidisciplinary resources.

2. Following established diagnostic and care pathways is becoming the norm; however, modification of care maps needs to be considered due to patient or disease-related factors.

3. Surgical decision-making includes setting realistic expectations on the part of the patients and their families, nant primary spinal neoplasms: anatomic considerations and operative nuances. Neurosurgery 2009;65(6, Suppl)173-181, discussion 181

38 Kawahara N, Tomita K, Baba H, et al. Cadaveric vascular anatomy for total en bloc spondylectomy in malignant vertebral tumors. Spine (Phila Pa 1976) 1996;21(12):1401-1407

39 Kawahara N, Tomita K, Murakami H, Demura S. Total en bloc spondylectomy for spinal tumors: surgical techniques and related basic background. Orthop Clin North Am 2009;40(1):47-63, vi

40 Lastra-Power J, Gokaslan ZL, Sosa IJ. Primary bony spinal lesions: surgery for cure. In: Benzel EC, ed. Spine Surgery: Techniques, Complication, Avoidance, and Management. Philadelphia, PA: Elsevier; 2005:1015-1024

41 Tomita K, Kawahara N, Murakami H, Demura S. Total en bloc spondylectomy for spinal tumors: improvement of the technique and its associated basic background. J Orthop Sci 2006; 11(1):3-12

including discussion of the expected high-rate of complications and the need for long-term follow-up.

4. As more detailed insights into the genomics of such neoplastic lesions emerge and more targeted pharmacologic treatments become available, the opportunity for large-scale data repositories to provide game-changing insights becomes very apparent. Fortunately, the AOSpine Neoplasia Knowledge Forum provides such a border- and specialty-defying platform for the engagement of surgeons at all levels of depth of practice exposure.

As always, EBSJ welcomes comments from its readership on the management and controversies surrounding spinal GCT management. 